\title{
Design and Modeling of a Radiofrequency Coil Derived from a Helmholtz Structure
}

\author{
Sidi M. Ahmed Ghaly \\ Electrical Engineering Department, \\ College of Engineering, Al Imam \\ Mohammad Ibn Saud Islamic \\ University, Riyadh, Saudi Arabia \\ smghaly@imamu.edu.sa
}

\author{
Khalid. A. Al-Snaie \\ Electrical Engineering Department, \\ College of Engineering, Al Imam \\ Mohammad Ibn Saud Islamic \\ University, Riyadh, Saudi Arabia \\ kalsnaie@imamu.edu.sa
}

\author{
Asad. M. Ali \\ Electrical Engineering Department, \\ College of Engineering, Al Imam \\ Mohammad Ibn Saud Islamic \\ University, Riyadh, Saudi Arabia \\ asadali@imamu.edu.sa
}

\begin{abstract}
This paper focuses on radiofrequency (RF) coils that can produce a high electromagnetic field homogeneity to be used for magnetic resonance imaging (MRI) applications. The proposed structure is composed of four wire loops symmetrically located on an ellipsoidal surface. The main objective of this work is to improve field homogeneity compared to a standard Helmholtz coil. Numerical simulation was carried out to assess the RF electromagnetic field behavior of the proposed coil. Different electrical modeling and simulations were investigated, particularly the study of the whole modeling of the proposed structure taking into account all the couplings between the loops. The proposed coil was evaluated and compared with the standard Helmholtz coil. Simulation and experimental results confirmed the good performance of the developed coil in terms of electromagnetic field homogeneity, efficiency, sensitivity, and quality factor.
\end{abstract}

Keywords-radiofrequency coils; modeling; simulation; design; electromagnetic field homogeneity; impedance measurements; MRI

\section{INTRODUCTION}

Since the demonstration of the nuclear magnetic resonance (NMR) phenomenon in 1946, the number of its applications grows steadily [1-3]. NMR imaging is based on the study of the magnetization modifications of the nuclei of a subject substance to a precise magnetic environment [4], in particular, a very strong magnetic field. The field homogeneity is needed over the observed area and it has been shown that the quality of the image obtained is, in particular, a function of the intensity of the applied static magnetic field and the homogeneity of the radiofrequency electromagnetic field produced by the transmitting coil and associated with the receiving coil [5-7]. The electromagnetic field homogeneity of radiofrequency coils is usually better with multiple radiating elements, of identical or different attributes [8,9], and their design can be accordingly optimized. For example, birdcage coils [8] are the most popular in MRI, they show outstanding performance in terms of magnetic field and signal-to-noise ratio (SNR), and have a sufficient number (usually greater than 8) of parallel radiating strands, often periodically placed on the surface of a cylinder. The optimization of RF coils is the subject of continuous research. Currently, inductive coupling problems between radiating elements (conductors) are largely taken into account, and this task is partly facilitated for periodic structures [10-12]. For RF coils that do not have a specific periodicity, optimization and implementation are more challenging. This is the case of Helmholtz coils [13] which consist of two coaxial circular coils separated by a distance equal to their radius. An improvement in the homogeneity of the field passes necessarily by the addition of coaxial turns. Thus, original structures, generally contained in a spherical and ellipsoidal envelope and having four or more free element loops have already been proposed [10-12] and very interesting properties in terms of homogeneity and sensitivity are obtained. A certain number of possibilities nevertheless remain to be analyzed. This research work considers aspects related to electronic instrumentation and in particular, the design, modeling and optimization of RF coils for magnetic resonance imaging, presenting a certain simplicity of implementation and having good homogeneity of RF electromagnetic field. More precisely, the optimization and modeling of a symmetrical coil with four coaxial circular loops producing effective sixth order homogeneity of electromagnetic field are presented. In fact, the proposed structure includes only two coaxial circuits. In NMR imagery, we have already demonstrated the advantages of the free element four-coil system $[10,11]$ that provides fourth order homogeneity. In this work, the main objective is to increase the sensitivity of the coil and reduce the number of tuned loops from four to two, which can be done by connecting the two loops positioned in the same side in series with a shared tuning capacitance. This configuration looks like a distributed Helmholtz coil.

This work, which includes modeling, simulations and experimental results, intends to be an important input for RF coils resulting from Helmholtz structure which can be used for MRI applications.

\section{ELECTRICAL MODELING}

In this section the electrical modeling of the proposed coil that includes all inductive and capacitive couplings will be discussed. The electrical modeling of the structure is necessary to demonstrate its operation and feasibility. The proposed structure is shown schematically in Figure 1. The proposed structure has the advantage to reduce the number of tuned 
circuits from four to two by connecting the two loops positioned in the same side with a shared tuning capacitance for each single circuit (two series circuits). This choice facilitates the comparison with the preceding proposed structures $[10,11]$. Thus, the developed coil looks like a distributed Helmholtz coil. To confirm the feasibility of such a coil configuration, a whole electrical model of the four-loop system compelling all capacitive and inductive couplings is investigated. The coupling mode of the rings is an inductive coupling [14] between all loops and a capacitive coupling [15] between adjacent left or right loops (connected in series). The equivalent electrical diagram of the system in the ideal case (without losses) is shown in Figure 2.

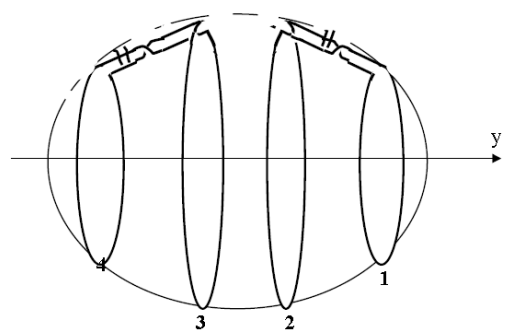

Fig. 1. Structure of the proposed coil

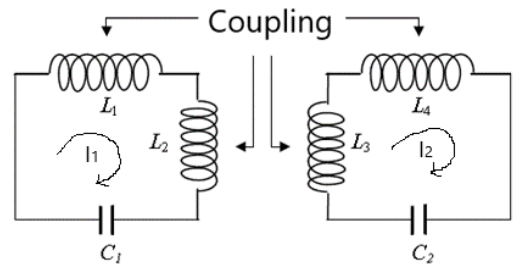

Fig. 2. Electrical diagram for the proposed coil (without losses)

Because of the probe symmetry, there are some obvious simplifications: Inductances $L_{4}=L_{1}, L_{3}=L_{2}$, capacitances $C_{1}=C_{2}$, mutual inductances $M_{34}=M_{12}$ and $M_{24}=M_{13}$. As for the Helmholtz coils [13] and using Kirchoff s voltage law, the two electrically isolated circuits crossed by the currents $I_{l}$ and $I_{2}$ are characterized by the following equation system:

$$
\left[\begin{array}{cc}
Z & j\left(M_{14}+M_{23}+2 M_{13}\right) \omega
\end{array}\right]\left[\begin{array}{l}
I_{1} \\
I_{2}
\end{array}\right]=\left[\begin{array}{l}
0 \\
0
\end{array}\right]
$$

Two modes of resonance can be distinguished: a co-current symmetrical mode with $I_{I}=I_{2}$, which is the mode of interest and a mode counter current which is not symmetric with $I_{l}=-I_{2}$ (gradient mode). For the co-current mode (the mode of interest), (1) can be reduced to:

$$
Z I_{1}+j\left(M_{14}+M_{23}+2 M_{13}\right) \omega I_{2}=0
$$

Introducing the coupling coefficients $k_{i j}[16]$, the resonance equation becomes:

$$
\begin{aligned}
& C\left(L_{1}+L_{2}+2 k_{12} \sqrt{L_{1} L_{2}}+\right. \\
& \left.2 k_{13} \sqrt{L_{1} L_{2}}+k_{14} L_{1}+k_{23} L_{2}\right) \omega^{2}=1
\end{aligned}
$$

Thus, it has a real positive solution:

$$
f_{1}=\frac{1}{2 \pi \sqrt{C\left(L_{1}+L_{2}+2 k_{12} \sqrt{L_{1} L_{2}}+2 k_{13} \sqrt{L_{1} L_{2}}+k_{14} L_{1}+k_{23} L_{2}\right)}}(4)
$$
of:

For the counter-current mode $\left(I_{l}=-I_{2}\right)$, there is a frequency

$$
f_{2}=\frac{1}{2 \pi \sqrt{C\left(L_{1}+L_{2}+2 k_{12} \sqrt{L_{1} L_{2}}-2 k_{13} \sqrt{L_{1} L_{2}}-k_{14} L_{1}-k_{23} L_{2}\right)}}(5)
$$

As for the Helmholtz coil, it is necessary to ensure sufficient decoupling of the modes by separating the loops sufficiently.

\section{OPTIMIZATION OF THE FIELD HOMOGENITY}

For the calculation of the electromagnetic field $\left(B_{1}\right)$ produced by the proposed structure which consists of two symmetric elements and has small dimensions compared to the wavelength, the superposition principle can be applied to obtain the total electromagnetic field [17]. The component along the $y$ axis of the magnetic field shown in Figure 1 produced by a thin ring of wire, of radius a, positioned in the $x z$ plane, centered on the origin $O$ and carrying a current $I$ is easily determined by using Biot-Savart's law [18]:

$$
B_{1 y}=\frac{\mu_{0} \mathrm{I}}{2 \alpha\left(1+\left[\frac{y}{\alpha}\right]^{2}\right)^{\frac{3}{2}}}
$$

The extension to the proposed structure permits to obtain the total field $B_{l y}$ along the coil axis as shown:

$$
B_{1 y}=\mu_{\mathrm{o}} I \sum_{i}^{4} \frac{1}{2 \alpha_{i}\left(1+\left[\frac{y-y_{i}}{\alpha_{i}}\right]^{2}\right)^{\frac{3}{2}}}
$$

where $y_{i}$ is the ordinate of the position of the loop $i$ on the axis and $\alpha_{i}$ is the radius of the loop (i).

Due to the symmetry of the structure, $B_{l y}$ component can be expressed as a Taylor expansion around the origin [19]. The odd orders of the function $B_{l y}$ should be zero and the Taylor's expansion gives the even. For a given ellipsoidal structure, the symmetry returns the computation parameters to two and thus, the spacing between the loops (inner or outside rings) or the diameter of the loops can be adjusted. The angles $\alpha_{i}$ can be introduced with respect to the axis (Oy), i.e. for loops 1 and 2, angles $\alpha_{1}$ and $\alpha_{2}\left(\alpha_{1}<\alpha_{2}\right)$ and for loops 3 and 4 , angles $\pi-\alpha_{2}$ and $\pi-\alpha_{1}$ respectively as shown in (8). It should be noted that the field calculation depends only on the geometry of the coils.

$$
\begin{gathered}
B_{1 y}=2 I \mu_{\mathrm{o}}\left\{\left(\frac{\sin \alpha_{1}^{3}}{a_{1}}\right)+\left(\frac{\sin \alpha_{2}^{3}}{a_{2}}\right)+\left(\left(\frac{\sin \alpha_{1}^{2}}{a_{1}}\right) P_{91}\left(\cos \alpha_{1}\right)\right)\left(\frac{y}{r_{1}}\right)^{8}+\right. \\
\left.\left(\left(\frac{\sin \alpha_{2}^{2}}{a_{2}}\right) P_{91}\left(\cos \alpha_{2}\right)\right)\left(\frac{y}{r_{2}}\right)^{8}+. .\right\}
\end{gathered}
$$

Indeed, on the electrical level, the transformation ratio between currents is forced to one and it is possible to modify the solution by simply scaling factor. After the numerical calculations, there is a certain range of possibilities for fourth order homogeneity field which advantageously presents a unique and optimal six-order solution for the proposed coil. There is a unique solution for which the six-order derivative of 
the electromagnetic field $\left(B_{1}\right)$ is zero. For this optimal case, the secondary geometric data for the proposed coil are given in Table I.

TABLE I. SECONDARY GEOMETRIC DATA FOR THE PROPOSED COIL

\begin{tabular}{|c|c|c|c|}
\hline Main axis $a(\mathrm{~cm})$ & Secondary axis $\boldsymbol{b}(\mathbf{c m})$ & $\alpha_{1}(\mathbf{r a d} / \mathbf{d e g})$ & $\alpha_{2}(\mathbf{r a d} / \mathbf{d e g})$ \\
\hline 3.042 & & $0.734 / 42.09$ & $1.299 / 74$ \\
\hline
\end{tabular}

\begin{tabular}{|l|l|l|l|}
\hline 3.042 & 2.459 & $0.734 / 42.09$ & $1.299 / 74.42$ \\
\hline
\end{tabular}

The eccentricity $e$ is given by:

$$
e=\sqrt{1-\left(\frac{b^{2}}{a^{2}}\right)}=0.587
$$

According to Table I, the Taylor expansion in (8) can be reduced to:

$$
B_{i y}=\left(I \mu_{\mathrm{o}} / 2\right)\left\{106.3-1.24710^{15} y^{8}+\cdots\right\}
$$

Figure 3 shows the obtained dimensions of the ellipsoidal coil and the corresponding normalized electromagnetic field profile:

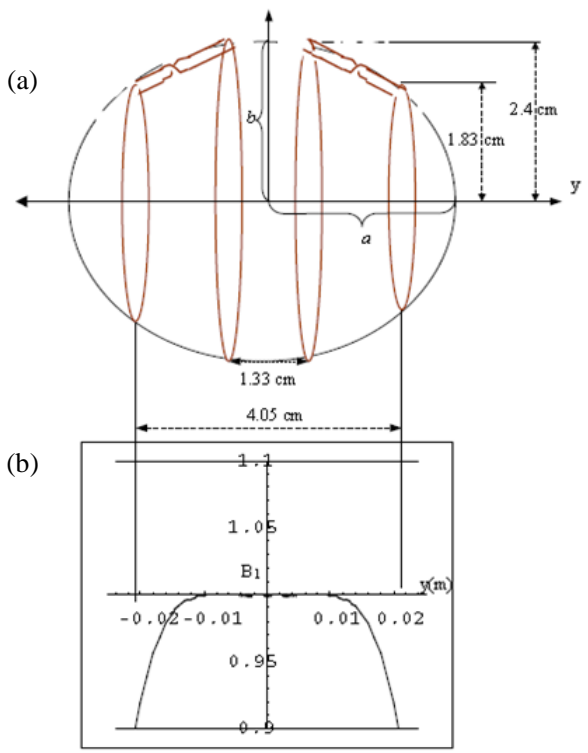

Fig. 3. (a) Dimensions of the proposed structure, (b) Normalized field profile

It is clear from Figure 3(b) that profile widths at $1 \%$ and $10 \%$ can be calculated and compared with those obtained from Helmholtz coil shown in Table II.

TABLE II. PROFILE WIDTHS AT 1\% AND 10\% FOR BOTH COILS (HELMHOLTZ AND PROPOSED)

\begin{tabular}{|c|c|c|}
\hline Structure & Profile width at 1\%(cm) & Profile width at 10\%(cm) \\
\hline Helmholtz & 1.50 & 2.89 \\
\hline Proposed & 2.92 & 4.44 \\
\hline
\end{tabular}

By comparison with Helmholtz coil, the proposed structure has a significant increase in the profile. Thus, in considering uniformity of $1 \%$, profile width has increased approximately $97 \%$ and at $10 \%$ uniformity the profile width increased $58 \%$ more than the Helmholtz coil. The performance described above is very general and does not depend on the size of the coils. Indeed, it suffices to apply a simple scale factor to the ellipsoidal structure to obtain coils with the same performances.

\section{Simulation}

In this part, the electrical behavior of the system will be investigated for the proposed structure (ellipsoidal coil). The system comprises of four coaxial wires with inductive coupling between all loops and capacitive coupling between adjacent left or right loops. It consists of two identical tuned circuits resonant at the same frequency.

\section{A. One Tuned Circuit Having Two Rings Coupled in Series}

In this case, assume a circuit tuned at frequency $f_{0}$ and consisting of two rings with inductances $L_{1}$ and $L_{2}$ mutually coupled as shown in Figure 4.

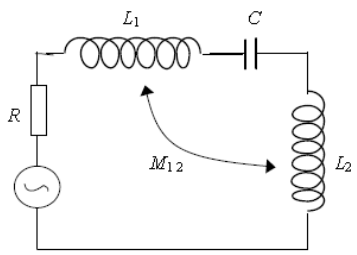

Fig. 4. Two mutually coupled inductances

In the case without losses, the value of the self-resonance frequency is given by:

$$
f_{0}=\frac{1}{2 \pi \sqrt{\left(L_{1}+L_{2}+2 k_{12} \sqrt{L_{1} L_{2}}\right) C}}
$$

\section{B. Case of the Proposed Structure (2 Tuned Circuits):}

Coaxial wire loops are characterized by their selfinductance and their mutual inductances, which depend at high frequencies on the geometrical configuration: loop diameter, wire diameter, and distances between the loops [20]. The two tuning capacitors are supposed to be identical. If we consider that the proposed structure is sourced by an ideal voltage source, then the equivalent electrical diagram is shown in Figure 5.

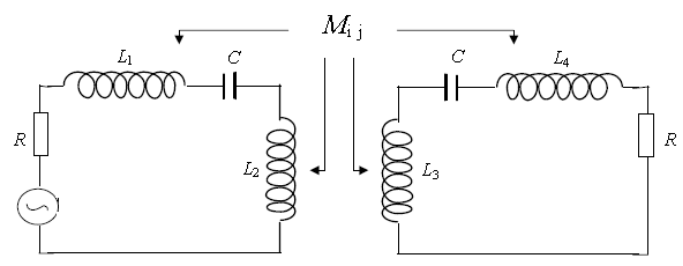

Fig. 5. Electrical diagram for the proposed coil (with losses)

The tuning capacitor is determined from the established formula of the resonance frequency $f_{l}$ of the coil as shown in (2). For the co-current mod, the tuning capacitor is deduced as:

$$
C=\frac{1}{2 \pi^{2} f_{1}{ }^{2}\left(L_{1}+L_{2}+2 k_{12} \sqrt{L_{1} L_{2}}+2 k_{13} \sqrt{L_{1} L_{2}}+k_{14} L_{1}+k_{23} L_{2}\right)}
$$

The resonance frequency $f_{l}=100.24 \mathrm{MHz}$ is the Larmor frequency at static magnetic field $B_{0}=3$ T [7]. Table III presents 
the data necessary for the preliminary adjustment of the ellipsoidal structure obtained from the equivalent electrical model in Figure 5. Tuning capacitor $C$ and self-resonance frequency $f_{0}$ of tuned circuits are presented in Table IV.

TABLE III. ELECTRICAL PARAMETERS AND TUNING ELEMENTS

\begin{tabular}{|c|c|c|c|c|c|c|}
\hline & \multicolumn{5}{|c|}{ Parameters (inductances $\boldsymbol{L}_{i}$ coupling $\boldsymbol{k}_{i j}$ ) } \\
\hline & $\boldsymbol{L}_{\boldsymbol{1}}=\boldsymbol{L}_{\mathbf{4}}$ & $\boldsymbol{L}_{\mathbf{2}}=\boldsymbol{L}_{\mathbf{3}}$ & $\boldsymbol{k}_{\boldsymbol{1 2}}$ & $\boldsymbol{k}_{13}$ & $\boldsymbol{k}_{14}$ & $\boldsymbol{k}_{23}$ \\
\hline Proposed Structure & 83.3 & 117.6 & 0.16 & 0.06 & 0.02 & 0.20 \\
\hline
\end{tabular}

TABLE IV. TUNING ELEMENTS

\begin{tabular}{|c|c|}
\hline $\boldsymbol{f}_{\boldsymbol{0}}$ & $\boldsymbol{C}$ \\
\hline $108.37 \mathrm{MHz}$ & $9.269 \mathrm{pF}$ \\
\hline
\end{tabular}

The simulation results give the quality factor of the proposed structure which equals to 305 with losses when $R_{1}=R_{2}=0.82 \Omega$. Figure 6 presents the simulation of the input impedance curve of the proposed structure. As seen, there are two resonance series: at $100 \mathrm{MHz}$ and $123 \mathrm{MHz}$. As the quality factor is high, then the energy absorbed at $f_{0}$ is low. On the other hand, since $R$ is small, the energies absorbed at $f_{1}$ and $f_{2}$ are relatively large [7]. For the resonance frequencies, Table V compares the simulation results with the theoretical (without losses) for the ellipsoidal coil.

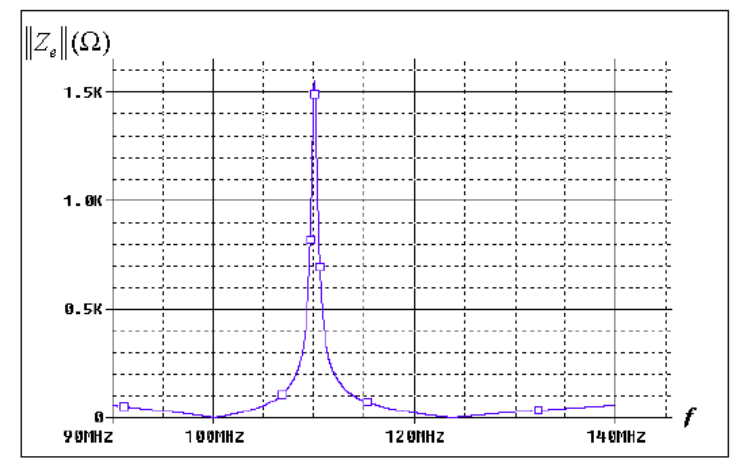

Fig. 6. Simulation of the input impedance vs frequency curve for the ellipsoidal coil.

TABLE V. SIMULATION AND THEORETICAL RESULTS WITHOUT LOSSES

\begin{tabular}{|c|c|c|}
\hline & \multicolumn{2}{|c|}{ Ellipsoidal structure } \\
\hline Modes of resonance & 1 & 2 \\
\hline Theoretical $\mathbf{f}_{\mathbf{1}}$ (Mhz) & 100.24 & 118.85 \\
\hline Simulation $\mathbf{f}_{\mathbf{1}}$ (Mhz) & 100.23 & 118.98 \\
\hline
\end{tabular}

A simulation of the currents variations in the ellipsoidal coil which includes two circuits only (carrying currents $I_{c 1}$ and $I_{c 2}$ ) is given in the Figure 7. It should be noted that at the two resonance frequencies $f_{1}$ and $f_{2}$, the currents $I c_{1}$ and $I c_{2}$ have maximum magnitude (almost identical) and the difference between the two modes remains essentially at the phase level: in-phase currents for $f_{1}$ and in opposition of phase for $f_{2}$.

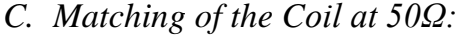

The proposed coil is matched using an inductive coupling [14]. This kind of matching uses an attack loop with the same dimensions as the first loop of the proposed coil and this attack loop is tuned to $f_{l}$ as shown in Figure 8 .

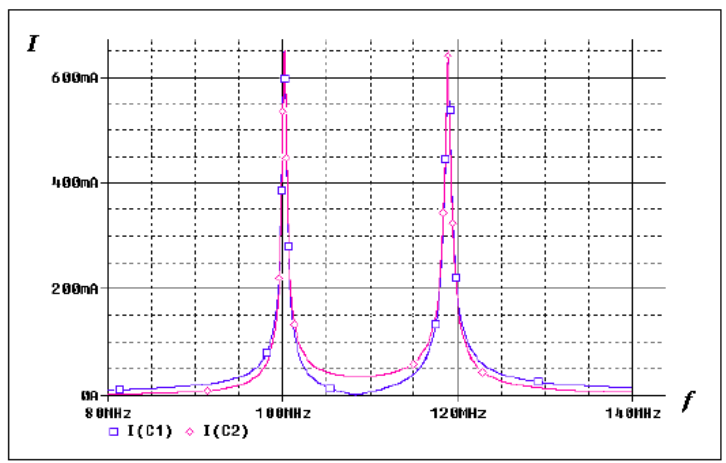

Fig. 7. Simulation of the currents variation vs frequency for the ellipsoidal coil.

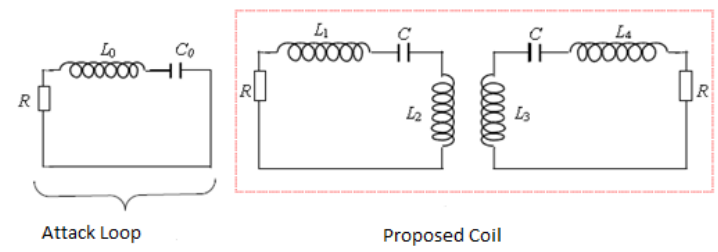

Fig. 8. Matching of the ellipsoidal coil with inductive coupling

The attack coil is inductively coupled with the four loops of the structure. The matching of the coil at $50 \Omega$ is performed by optimizing the coupling loop position. The associated coupling coefficients $\left(k_{0 i}, i=1, . ., 4\right)$ allowing the matching are presented in Table VI. The simulation of the matching at $50 \Omega$ (Figure 9) gives a corresponding distance between the attack loop and the ellipsoidal coil equal to $2.56 \mathrm{~cm}$. The inductive coupling results in an impedance inversion: the series resonance modes become parallel.

TABLE VI. ASSOCIATED COUPLING COEFFICIENTS

\begin{tabular}{|c|c|c|c|c|}
\hline Coupling $\boldsymbol{k}_{i j}$ & $\boldsymbol{k}_{\boldsymbol{0 1}}$ & $\boldsymbol{k}_{\boldsymbol{0} 2}$ & $\boldsymbol{k}_{\mathbf{0 3}}$ & $\boldsymbol{k}_{\boldsymbol{0} 4}$ \\
\hline Ellipsoidal structure & 0.0736 & 0.0216 & 0.0096 & 0.0018 \\
\hline
\end{tabular}

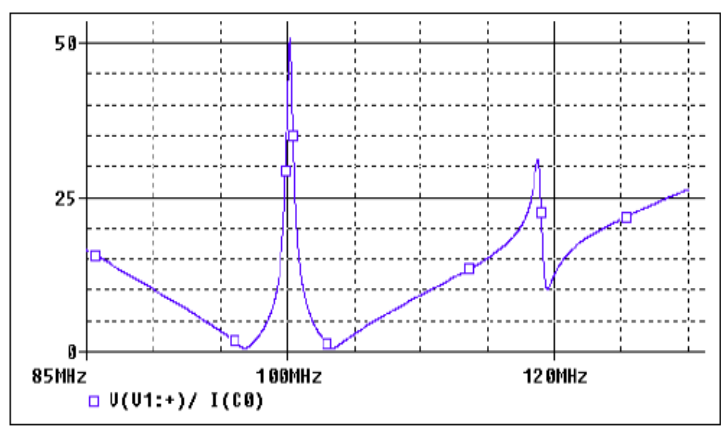

Fig. 9. Magnitude of the input impedance vs frequency.Simulation of the matching for the ellipsoidal coil at $f_{l}=100 \mathrm{MHz}, R=50 \Omega$. 


\section{EXPERIMENTAL PART}

In this part, we present the experimental results obtained with the developed coils (Helmholtz and ellipsoidal). We conducted out-of-field tests using a network analyzer. Relative measurements of the RF electromagnetic field $\left(B_{l}\right)$ along the main axis of the coils were carried out and were compared with those found in theory.

\section{A. Prototype Dimensions}

To facilitate comparisons, both standard Helmholtz coil and the proposed (Figure 10) had the same radial diameter $d$ equal to $4.80 \mathrm{~cm}$ (Table VII).

TABLE VII. COIL DIMENSIONS

\begin{tabular}{|c|c|c|c|c|}
\hline & \multicolumn{2}{|c|}{ Diameter } & \multicolumn{2}{c|}{ Distances } \\
\hline Structure & Inner loops & Outer loops & Inner loops & Outer loops \\
\hline Helmholtz & 4.80 & -- & 2.40 & -- \\
\hline Ellipsoidal & 4.80 & 3.60 & 1.33 & 4.05 \\
\hline
\end{tabular}

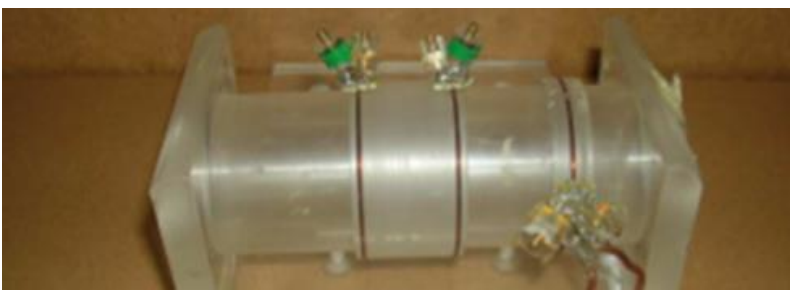

(a)

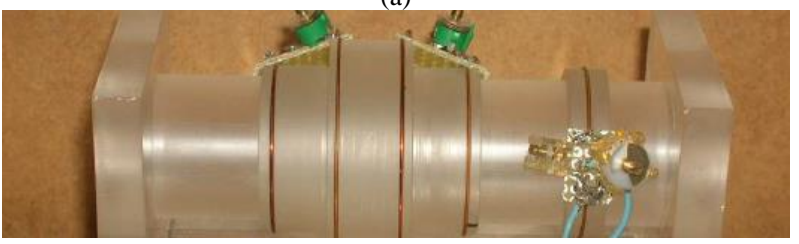

(b)

Fig. 10. The developed (a) Helmholtz and (b) ellipsoidal coils

\section{B. Matching and Tuning of the Coils}

It is possible to proceed directly to the tuning of the coils, but it requires a certain control of the various influential parameters (stepwise adjustment of the tuning capacitors). The tuning of each loop at $f_{0}$ is performed individually by shorting the other loops and bringing it closer to a test loop. By predetermination of $f o$ (see (4)), a vacuum presetting of the coil is carried out. Figure 11 gives an explanatory diagram.

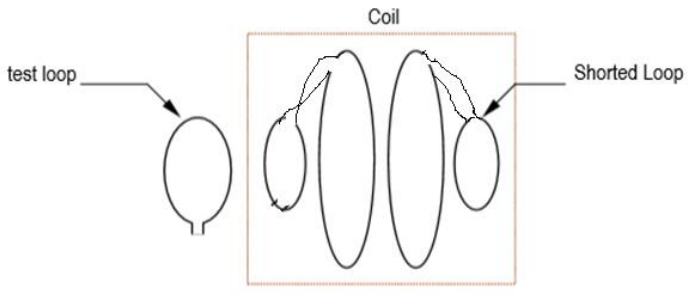

Fig. 11. Individual tuning of ellipsoidal coil

Figure 12 shows the variations of the module and phase of the input impedance seen by the source as a function of the frequency.

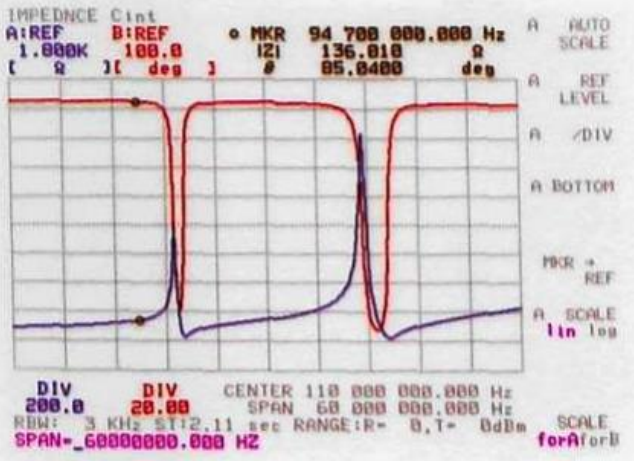

Fig. 12. Photograph of the tuning of the ellipsoidal coil

Two parallel resonance frequencies are observed for the ellipsoidal coil. Table VIII gives the comparison between the values of the experimental measured frequencies (with losses) and those in theory (without losses) for the ellipsoidal coil:

TABLE VIII. EXPERIMENTAL AND THEORETICAL VALUES

\begin{tabular}{|c|c|c|}
\hline \multicolumn{3}{|c|}{ Ellipsoidal coil } \\
\hline Resonance mode $\boldsymbol{i}$ & 1 & 2 \\
\hline $\boldsymbol{f}_{\boldsymbol{i}}$ theoretical $(\mathbf{M H z})$ & 100.25 & 118.85 \\
\hline $\boldsymbol{f}_{\boldsymbol{i}}$ experimental $(\mathbf{M H z})$ & 100.24 & 117.91 \\
\hline
\end{tabular}

It can be observed that we have almost the same value of the frequency $f_{l}$, while there is a small error for the other frequencies. For the last mode of resonance, the error is almost $3 \%$ for the ellipsoidal coil. Impedance matching allows optimal energy transfer between coils and the transmission and reception system [14]. For optimal transfer in the coaxial transmission cable during excitation, the impedance of the system should be $50 \Omega$ [14]. The matching of the coils can be realized, either using capacitors (capacitive coupling) or using a coupling loop (inductive coupling). In our case, we opted for the matching by inductive coupling because it allows having a electrically balanced circuit. The matching of the coil is achieved by optimizing the position of the attack loop with the coil. Thus, the value of the distance to have an impedance of $50 \Omega$ is equal to $1.9 \mathrm{~cm}(1.91 \mathrm{~cm}$ in theory) for the ellipsoidal coil. The impedance curve obtained for the matched ellipsoidal coil with impedance measurements using Network Analyzer is given in Figure 13.

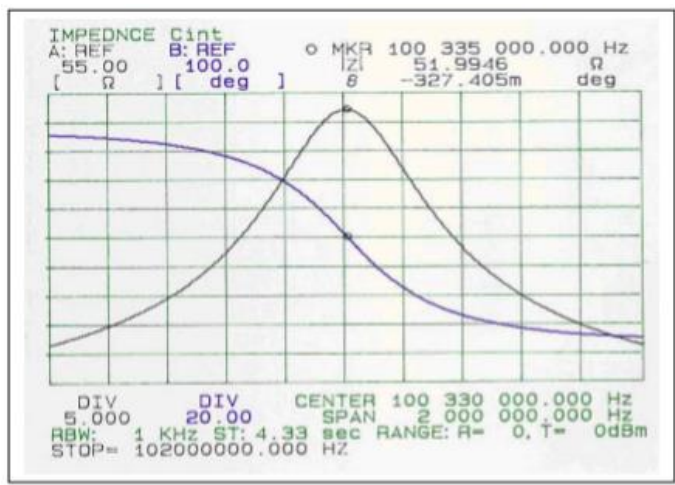

Fig. 13. Impedance curve obtained for the matched ellipsoidal coil 


\section{Results}

Comparative tests have been carried out between the ellipsoidal and Helmholtz coils. Thus, relative field measurements along the axis of the coils were made using a small test loop connected to the Network Analyzer. Figure 14 shows the measurement bench. We were able to take the electromagnetic field along the axis of the coils (Oy). The curves of this normalized field produced by both coils are given in Figure 15.

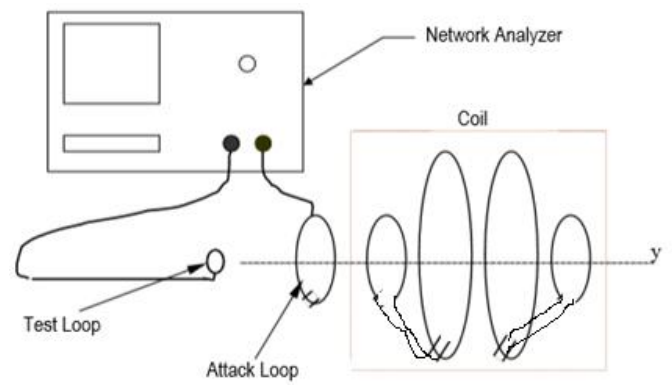

Fig. 14. The measurement bench

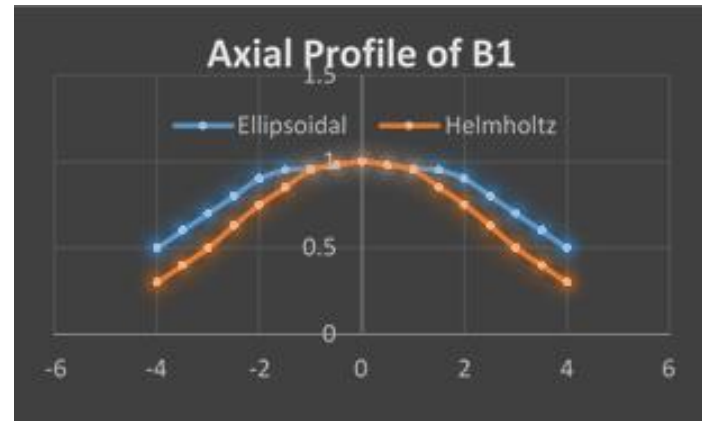

Fig. 15. The curves of the normalized field produced by both coils

To compare the axial profiles, we determined the relative widths of the lobe at $10 \%$. Thus, compared to the Helmholtz coil, the relative width of the lobe at $10 \%$ is 1.54 times larger for the ellipsoidal coil (see Table IX), which is in accordance with the theoretical forecasts (Table II). Similarly, for the same absorbed power, it is possible to check the efficiency of the coils [22]. Compared to the Helmholtz coil, the center field is 1.42 times larger for the ellipsoidal coil as shown in Table IX. By scanning with frequency and using two sufficiently distant coils (one transmitting and the other receiving), an improvement of the quality factor of $47 \%$ for the ellipsoidal coil is noted. It is increased from 215 for the Helmholtz coil to 316 for the ellipsoidal coil (Table IX).

TABLE IX. COIL PERFORMANCE COMPARISON

\begin{tabular}{|c|c|c|}
\hline Coil & Helmholtz & Ellipsoidal \\
\hline Relative widths of lobe at $\mathbf{1 0 \%}$ & 1 & 1.54 \\
\hline Efficency & 1 & 1.42 \\
\hline Quality factor & 215 & 316 \\
\hline
\end{tabular}

\section{CONCLUSION}

We presented the design and testing of a prototype magnetic resonance imaging coil composed of two free distributed elements contained in an ellipsoidal envelope and connected directly in series via a tuning capacitor. Compared to classic Helmholtz coils, the goal was to improve the homogeneity of the RF electromagnetic field produced by the coil to get an effective sixth order homogeneous field. A whole complete electrical modeling of the prototype coil was investigated, taking into account all couplings (inductive and capacitive). For a given resonance frequency, predetermination of the tuning frequency of the coil's elements is performed, as well as the calculation of the associated tuning capacitors. The matching of the prototype coil by inductive coupling allowed great flexibility for settings and a real simplicity of design.

Comparative tests and measurements between a reference Helmholtz coil and the prototype coil with the same radial diameter, have been carried out and were in good agreement with the theoretical predictions. Thus, the observed width of the field homogeneity lobe at $10 \%$ is considerably increased for the proposed coil. A significant improvement in the quality factor was also observed for the ellipsoidal coil. Moreover, for the developed ellipsoidal coil, efficiency and signal-to-noise ratio are higher as well. These good performance values for the ellipsoidal prototype coil give motivation to test the coil with load (with real samples) for MRI applications.

\section{ACKNOWLEDGMENT}

Authors would like to thank the deanship of Academic research at Al Imam Mohammad Ibn Saud Islamic University for the financial support of the project: No 371411/1437H.

\section{REFERENCES}

[1] F. Remeo, D. I. Hoult, "Magnet field profiling: Analysis and correcting coil design”, Magnetic Resonance in Medicine, Vol. 1, No. 1, pp. 44- 65, 1984

[2] C. E. Hayes, W. A. Edelstein, J. F. Schenck, O. M. Mueller, M. Eash, "An efficient, highly homogeneous radiofrequency coil for whole-body NMR imaging at 1.5 T", Journal of Magnetic Resonance, Vol. 63, No. 3, pp. 622-628, 1985

[3] J. T. Vaughan, H. P. Hetherington, J. O. Out, J. W. Pan, G. M. Pohost, "High frequency volume coils for clinical NMR imaging and spectroscopy", Magnetic Resonance in Medicine, Vol. 32, No. 2, pp. 206-218, 1994

[4] K. Asher, N. K. Bangerter, R. D. Watkins, G. E. Gold, "Radiofrequency Coils for Musculoskeletal MRI", Topics in Magnetic Resonance Imaging, Vol. 21, No. 5, pp. 315-323, 2010

[5] Q. X. Yang, S. Li, M. B. Smith, "A Method for Evaluating the Magnetic Field Homogeneity of a Radiofrequency Coil by Its Field Histogram", Journal of Magnetic Resonance, Series A, Vol. 108, No. 1, pp. 1-8, 1994

[6] S. Li, Q. X. Yang, M. B. Smith, "RF coil optimization: Evaluation of B1 field homogeneity using field histograms and finite element calculations", Magnetic Resonance Imaging, Vol. 12, No. 7, pp. 10791087,1994

[7] B. Gruber, M. Froeling, T. Leiner, D. W. J. Klomp, "RF coils: A practical guide for nonphysicists", Journal of Magnetic Resonance Imaging, Vol. 48, No. 3, pp. 590-604, 2018

[8] J. Mispelter, M. Lupu, A. N. M. R. Briguet, NMR Probeheads for Biophysical and Biomedical Experiments: Theoretical Principles \& Practical Guidelines, Imperial College Press, 2006

[9] B. J. Dardzin, S. S. Li, C. M. Collins, G. D.Williams, M. B. Smith, "A Birdcage Coil Tuned by RF Shielding for Application at 9.4 T", Journal of Magnetic Resonance, Vol. 131, No. 1, pp. 32-38, 1998

[10] S. M. A. Ghaly, S. S. Al-Sowayan, "A high B1 field homogeneity generation using free element elliptical four-coil system", American Journal of Applied Sciences, Vol. 11, No. 4, pp. 534-540, 2014 
[11] S. M. A. Ghaly, K. A. Al-Snaie, S. S. Al-Sowayan, "Design and Testing of Radiofrequency Spherical Four Coils", Modern Applied Science, Vol. 10, No. 5, pp. 186-193, 2016

[12] S. M. A. Ghaly, K. A. Al-Snaie, O. K. Mohammad, "Spherical and Improved Helmholtz Coil with High B1 Homogeneity for Magnetic Resonance Imaging", American Journal of Applied Sciences, Vol. 13, No. 12, pp. 1413-1418, 2016

[13] J. Wang, S. She, S. Zhang, "An improved Helmholtz coil and analysis of its magnetic field homogeneity", Review of Scientific Instruments, Vol. 73, No. 5, pp. 2175-2179, 2002

[14] M. Decorps, P. Blondet, H. Reutenauer, J. P. Albrand, C. Remy, "An inductively coupled, series tuned NMR probe", Journal of Magnetic Resonance, Vol. 65, No. 1, pp. 100-109, 1995

[15] J. Murphy-Boesch, A. P. Koretsky, "An in vivo NMR probe circuit for improved sensitivity", Journal of Magnetic Resonance, Vol. 54, No. 3, pp. 526-532, 2003

[16] R. F. Lee, R. O. Giaquinto, C. J. Hardy, "Coupling and Decoupling Theory and Its Application to the MRI Phased Array", Magnetic Resonance in Medicine, Vol. 48, pp. 203-213, 2002

[17] C. M. Collins, Z. Wang, "Calculation of Radiofrequency Electromagnetic Fields and Their Effects in MRI of Human Subjects", Magnetic Resonance in Medicine, Vol. 65, No. 5, pp. 1470-1482, 2011

[18] Y. E. Esin, F. N. Alpaslan, "MRI image enhancement using Biot-Savart law at 3 tesla", Turkish Journal of Electrical Engineering \& Computer Sciences, Vol. 25, pp. 3381-3396, 2017

[19] D. M. Ginsberg, M. J. Melchner, "Optimum Geometry of saddle shaped coils for generating a uniform magnetic field", Review of Scientific Instruments, Vol. 41, No. 1, pp. 122-123, 2010

[20] H. Fujita, T. Zheng, X. Yang, M. J. Finnerty, S. Handa, "RF Surface Receive Array Coils: The Art of an LC Circuit", Journal of Magnetic Resonance Imaging, Vol. 38, No. 1, pp. 12-25, 2013 